\title{
Australia: no al Protocolo de Kyoto, sí a la Asociación Asia-Pacífico sobre Desarrollo Limpio y Cambio Climático
}

$\mathrm{E}$ 1 Protocolo de Kyoto, primer acuerdo internacional que tiene como objetivo reducir la emisión de gases de efecto invernadero, los cuales generan el calentamiento de la Tierra, entró en vigor a principios de 2005. La puesta en marcha de dicho convenio estuvo marcada por dos tipos de expectativas: las positivas y las negativas. Ambas posturas fueron emitidas al interior tanto de los organismos no gubernamentales como de los gobiernos nacionales y de la sociedad civil en su conjunto.

La causa de esta polaridad de opinión tiene su fundamento, por un lado, en la esperanza que despierta el funcionamiento de un acuerdo supranacional que pretende poner fin al cambio climático (con todos los desastres naturales, junto con las pérdidas humanas y económicas, que esto conlleva) $y$, por el otro, por la falta de compromiso por parte de Estados Unidos (el mayor emisor de dióxido de carbono en el mundo) y Australia (el segundo productor de carbón en el planeta), países desarrollados que dijeron no al Protocolo de Kyoto.

En su momento, la justificación de Estados Unidos para no ratificar el Acuerdo se basaba en la protección económica, pues, según el gobierno de Bush, los objetivos

\footnotetext{
* Investigadora del Departamento de Estudios del

Pacífico de la Universidad de Guadalajara.

ORCID http://orcid.org/0000-0002-4553-8141

34 MÉXICO YLACUENCADELPACÍFICO

vol. 8, núm. 25 / mayo-agosto de 2005
}

del Protocolo ponían en riesgo el sector económico de su país. Mientras, Australia argumentaba que, sin el compromiso expreso por parte del país más contaminante del mundo (Estados Unidos), y sin la obligatoriedad de China e India (países signatarios del Protocolo) de reducir sus emisiones de dióxido de carbono, ratificar el Acuerdo resultaba "casi inútil y perjudicial para un país como Australia". ${ }^{1}$

Pareciera que el razonamiento del gobierno australiano es simple o egoísta; sin embargo, es producto de una reflexión práctica y económica. Es decir, los incentivos son bajos cuando la nación que emite la cuarta parte de la emisión de gases de efecto invernadero decide no comprometerse, a la vez que a las economías emergentes, como China, e India, quienes juntas emitían el 18\% de las emisiones del mundo en 1998 (cifras muy elevadas si se considera que Australia para la misma fecha sólo contribuía con 1.4\%), no se las obliga (dentro del Protocolo) a reducir su emisiones.

Con tal acción, la opinión pública, aunque desaprobó la falta de solidaridad de Australia hacia un bien público mundial, de alguna manera entendió sus razones. Sin embargo, cinco meses después de la entrada en vigor del Protocolo de Kyoto, Australia, junto con Estados Unidos, empezó a promover la Asociación Asia-Pacífico sobre Desarrollo Limpio y Cambio Climático, la 
cual abandera como compromiso "trabajar por la erradicación del efecto invernadero, sin entorpecer el crecimiento económico". ${ }^{2}$

Esta noticia provocó un sinfín de reacciones; para algunos, Australia y Estados Unidos buscaban desviar la presión internacional por su negativa al Convenio; ${ }^{3}$ para otros, se trataba de un boicot o un desafío al Protocolo de Kyoto, ${ }^{4}$ mientras que unos pocos argüían que se trataba de un reforzamiento o un complemento al Acuerdo. ${ }^{5}$ No obstante la diversidad de opiniones, las dos economías promotoras están tratando de vender a la comunidad internacional la idea de que su propuesta es una solución más efectiva para el calentamiento de la Tierra que el Protocolo de Kyoto.

Aun con el revuelo anterior, en la primera mitad de enero de 2006 se celebró en la ciudad australiana de Sydney el primer encuentro de la Asociación Asia-Pacífico sobre Desarrollo Limpio y Cambio Climático. Empero, ¿qué significa lo anterior? ¿Existen dos Acuerdos que persiguen un mismo fin, bajo diferentes medios? ¿Por qué Australia dijo no al Protocolo de Kyoto y sí a este nuevo Acuerdo? ¿Qué elementos del convenio hacen suponer a Australia que es más efectivo que el Protocolo? ¿De qué países está compuesta la nueva propuesta? Y, por último, ¿se puede esperar que con el funcionamiento de los dos Acuerdos se ponga fin al calentamiento global de la Tierra?

Estas son las preguntas que a lo largo de este documento se tratarán de responder. En el primer apartado se abordará una pequeña introducción de la creación de ambos Acuerdos, luego se presentarán los objetivos y los medios que tiene cada uno de ellos para alcanzarlos. En el segundo, se realizará un análisis comparativo entre los dos convenios. Luego, con el desarrollo anterior y agregando algunas reflexiones de las ONG, se tratará de llegar a una conclusión sobre los motivos que tuvo Australia para crear la Asociación Asia Pacífico sobre Desarrollo Limpio y Cambio Climático.

\section{El Protocolo de Kyoto y el Acuerdo Asia-Pacífico sobre Desarrollo Limpio y Cambio Climático}

El proceso de la puesta en marcha del Protocolo de Kyoto fue tan complejo que tardó casi siete años para empezar a funcionar. Determinar detalles, realizar investigaciones, establecer acuerdos, negociar objetivos, más el convencimiento individual de cada uno de los países miembros de Naciones Unidas para ratificarlo, es una muestra de la difícil tarea que resulta echar a andar un proyecto que ponga freno al calentamiento global de la Tierra.

Cuando entró en vigor el Protocolo de Kyoto, el objetivo a que se comprometieron los 143 países signatarios fue reducir las emisiones de gases de efecto invernadero en el periodo 2008-2012, por lo menos en $5.2 \%$ con relación a los niveles de 1990 . $^{6}$ Sin embargo, los únicos países que están obligados a bajar sus cantidades emitidas son los pertenecientes al anexo I, ${ }^{7}$ economías en su mayoría industrializadas, los cuales, con sólo el $20 \%$ de la población mundial, son responsables del $60 \%$ de las emisiones totales.

Las estrategias complementarias que se propusieron fueron: la mitigación y la adaptación. La primera se refiere "a toda la intervención del hombre concebida para reducir las emisiones de gases de efecto invernadero en sus fuentes de origen o para incrementar los sumideros de carbono". La segunda, alude "a los ajustes en los sistemas naturales o humanos en respuesta a estímulos climáticos o a sus efectos, con el fin de disminuir el daño que ocasionan o 
aprovechar sus beneficios". ${ }^{8}$ Es decir, para mitigar los efectos del cambio climático se establecen dos vías. La primera es tomar acciones para disminuir la emisión de gases de efecto invernadero (en todo sistema que utilice para su funcionamiento la quema de combustible) o la creación de más sumideros de dióxidos de carbono (como pueden ser los bosques). Mientras que la segunda impulsa a adaptarse a los efectos de los cambios climáticos para aminorar el perjuicio.

Las dos estrategias conllevan, haciendo un análisis simple, una relación costo-beneficio. ${ }^{9}$ En la mitigación, tanto la industria interna de cada país como la ciudadanía en general tendrían que modificar el comportamiento de producción y

La Asociación
Asia-Pacífico sobre
Desarrollo Limpio y
Cambio Climático, mejor
conocida como AP6,
surgió como iniciativa de
los grandes desertores
del Protocolo de Kyoto:
Australia y Estados
Unidos
de consumo que han tenido hasta ahora (con las perdidas económicas, de empleo y de competitividad que ello trae consigo). Mientras, la adaptación propone a todos los sectores del país (agrícola, salud, construcción, educativo, financiero, industrial) estar atentos a las nuevas necesidades que acarrea el calentamiento global para prevenirlo y enfrentarse a ello de la mejor manera. En la primera, el beneficio es revertir los efectos del cambio climático y seguir gozando de los recursos naturales que se tienen en la actualidad, mientras que en la segunda es la sobrevivencia.

Considerando que las medidas internas para desarrollar la estrategia de mitigación resultan muy costosa a corto y mediano plazos, los propios miembros del Protocolo incluyeron los mecanismos flexibles: a) la aplicación conjunta, ${ }^{10}$ b) los mecanismos de desarrollo limpio ${ }^{11}$ y c) el comercio de los derechos de emisiones. ${ }^{12} \mathrm{Se}$ proponen dos acciones: 1 . Invertir en tecnología limpia en otros países, sean desarrollados o no desarrollados, con el fin de que la disminución, producto de la tecnología invertida, sea contada como reducción de emisiones del país inversor; 2. Que las economías que emitan gases contaminantes por debajo del límite impuesto por el Protocolo puedan vender sus sobrantes a aquellos países que los excedan.

Como se puede observar, estas medidas no solamente reducen los costos de las medidas internas, sino también permiten a los países del anexo I alcanzar el objetivo del Protocolo, pues, evidentemente, los proyectos de inversión tecnológica que se realizan en otros países resultan mucho más económicos que los que su propia industria requiere. $\mathrm{Al}$ mismo tiempo, comprar bonos de carbono representa reducciones internas, mientras que al país que vende el excedente le permite invertir en investigación científica para la creación de tecnología limpia.

Esta es, a grandes rasgos, la situación que engloba al Protocolo con sus objetivos y los medios para alcanzarlos. Ahora lo interesante será conocer la situación de la Asociación Asia-Pacífico sobre Desarrollo Limpio y Cambio Climático, pues ello permitirá valorar los motivos que tuvo Australia para crearla, al tiempo que encontraremos la factibilidad de esta nueva propuesta.

La Asociación Asia-Pacífico sobre Desarrollo Limpio y Cambio Climático, mejor 
conocida como AP6, surgió como iniciativa de los grandes desertores del Protocolo de Kyoto: Australia y Estados Unidos. A su vez, las voces de estas controversiales economías encontraron eco en los gobiernos de Japón, Corea del Sur, China e India, países, todos ellos, miembros del Protocolo de Kyoto.

Este proyecto, que inició su gestación en el verano del 2005, se concretó a principios de enero de 2006, fecha en que se ofreció a la opinión pública el plan de acción del Acuerdo. Durante este foro estuvieron presentes los ministros de los países mencionados, más los principales ejecutivos de las grandes compañías mineras y petroleras (Exxon Mobil, Río Tinto y Peabody Energy, entre otras).

Durante la cumbre inaugural, el ministro de energía de Australia, Ian MacFarlane, afirmó que "ha llegado la hora de que el sector privado asuma el liderazgo de la lucha contra la emisión de gases con efecto invernadero"; esta intervención fue reafirmada por el ministro de energía estadounidense, Samuel Bodman, quien agregó que "son el sector privado, las empresas, los propietarios de las infraestructuras, quienes finalmente resolverán el problema; mientras que el papel de los gobiernos debe ser facilitarles el trabajo, en vez de obligar a hacerlo". ${ }^{13}$

La propuesta de la AP6 es simple, pues pretende vencer al calentamiento global mediante la promoción de tecnologías no contaminantes; sin embargo, no entra en su plan obligar a reducir la emisión de gases de efecto invernadero. ${ }^{14}$ Es decir, el gobierno no va a pedir a la industria nacional producir menos, sino que, con la implementación de tecnología limpia, por cada unidad de producto bruto producida se emitirá una menor cantidad de gases de efecto invernadero. ${ }^{15}$ Con esta medida se pretende conjugar la relación de va- riables: menor impacto ecológico y mayor crecimiento económico.

Para justificar lo anterior, el primer ministro australiano, John Howard, señaló que "el mundo seguirá utilizando combustibles fósiles durante muchos años, porque es más económico hacerlo; por lo tanto, es de sentido común que se intente y se logre hacer más limpio el uso de los combustibles fósiles" ${ }^{16}$

Lo que queda claro en la AP6 es que la clave para reducir la emisiones de gases se apuesta en la tecnología. Por tal compromiso se determinó la creación de ocho grupos especiales, destinados a la investigación de tecnologías que no generen daños ecológicos. Por otro lado, también agregaron dar un mayor peso a las energías renovables, al tiempo que pronosticaron un incremento en la utilización de la energía nuclear y el almacenamiento de carbono y de metano. ${ }^{17}$

En este mismo encuentro, el ministro australiano, John Howard, señaló que su país destinará 75 millones de dólares estadounidenses en los próximos cinco años, para combatir el calentamiento global, mientras que el presidente del Consejo Medioambiental de la Casa Blanca, James Connaughton, señaló que Estados Unidos destinará 52 millones de dólares (en 2007) para gestionar el trabajo de este Acuerdo. ${ }^{18}$

Al finalizar esta cumbre, cinco aspectos fueron relevantes: 1. El énfasis en soluciones tecnológicas. 2. La responsabilidad directa del sector privado para reducir sus emisiones contaminantes. 3. El compromiso, a la par, de los países desarrollados y en desarrollo, en los proyectos no contaminantes..$^{19}$ 4. Que el acuerdo no impone a sus socios obligaciones firmes para disminuir los gases de efecto invernadero. 5. La utilización de energía nuclear para mitigar el flujo de gases contaminantes. 


\section{Análisis comparativos entre los dos Acuerdos}

Hasta aquí se presentaron los dos Acuerdos internacionales que aparentemente tienen el mismo fin; ahora lo interesante es analizar las diferencias y las semejanzas para poder entender, de alguna manera, por qué para Australia la AP6 es un mecanismo más convincente:

1. Para empezar, existe una enorme diferencia entre el número de países participantes en cada Asociación, pues el Protocolo lo componen casi todos los miembros de $\mathrm{Na}$ ciones Unidas, mientras que en la AP6 sólo son seis economías. Sin embargo, la conjunción de estas últimas es muy interesante, ya que, con el $46 \%$ de la población mundial, son los causantes de casi el 50\% de las emisiones totales. Es decir, en este conjunto de países hay una estrecha relación entre el número de la población y las cantidades de dióxido de carbono emitidas.

El cuadro 1 muestra un dato relevante, Australia, en emisiones per cápita está casi en las mismas circunstancias que el país más contaminante del mundo, Esta-

\section{Cuadro 1 \\ Asociación Asia-Pacífico sobre Desarrollo Limpio y Cambio Climático}

\begin{tabular}{lrcc}
\hline Países & $\begin{array}{c}\text { Población } \\
\text { total }\end{array}$ & \multicolumn{2}{c}{$\begin{array}{c}\text { Emisión de dióxido de } \\
\text { carbono }\end{array}$} \\
\cline { 2 - 4 } & $\begin{array}{c}\text { Millones } \\
\text { (2002) }\end{array}$ & $\begin{array}{c}\text { Per cápita } \\
\text { (toneladas } \\
\text { métricas) }\end{array}$ & $\begin{array}{c}\text { Porcentaje } \\
\text { del total } \\
\text { mundial }\end{array}$ \\
\hline Australia & 19.5 & 18 & 1.4 \\
Corea del Sur & 47.4 & 9.1 & 1.8 \\
China & $1,294.9$ & 2.2 & 11.5 \\
Estados Unidos & 291 & 19.8 & 23.1 \\
India & $1,049.5$ & 1.1 & 4.4 \\
Japón & 127.5 & 9.3 & 4.9 \\
Total Ap6 & $2,829.8$ & 59.5 & 47.1
\end{tabular}

Fuente: Informe Sobre Desarrollo Humano 2004, PNUD.

38

MÉXICO YLACUENCADEL PACÍFICO

vol. 8, núm. 25 / mayo-agosto de 2005 dos Unidos. Por otro lado, un hecho único ocurre con Japón, pues en cifras absolutas emite más del doble de contaminación que Corea del Sur, pero en cifras relativas (total de emisiones por número de habitantes), los dos países producen cifras similares de gases de efecto invernadero, aun cuando el país nipón tiene 80 millones más de habitantes. Por otro lado, Japón, con sólo el $12 \%$ de la población que tiene la India, dentro de los porcentajes de emisión total, genera casi la misma cantidad de dióxido de carbono. Con base en lo anterior, queda claro que la AP6 sí es una Asociación relevante en cuanto se refiere a la influencia sobre el cambio climático. En consecuencia, aunque es baja la cantidad de socios, es suficiente para echar a andar un proyecto de esta magnitud.

2. La AP6 pone énfasis en la tecnología para mitigar el efecto invernadero, pero el Protocolo de Kyoto también la considera como un medio. La diferencia entre los dos consiste en que la comisión organizadora del Protocolo es consciente de que el proceso de investigación científica, de innovación y desarrollo de tecnología, requiere de tiempos no determinados y de costos que de alguna manera afectan el precio de los productos finales, junto con la pérdida de competitividad en el mercado internacional que ello acarrea. Esto no quiere decir que dejan de lado la tecnología, sino que están conscientes de los tiempos; por tal razón se crearon los mecanismos flexibles, para que, con la tecnología que ya cuentan los países desarrollados, la apliquen en otras naciones que carecen de ella (mientras los países industrializados siguen innovando para emplearla en su propia industria). En este sentido, no existe una diferencia real entre las dos propuestas; lo que existe es más bien un análisis más profundo por parte del Protocolo de Kyoto. 
3. En cuanto a responsabilizar, de manera directa, a los dueños del capital con las reducciones de las emisiones y el establecimiento del papel del gobierno como facilitador, también se tienen observaciones. Se entiende la postura porque, evidentemente, los principales daños ecológicos provienen del sector industrial. No obstante, partamos del hecho de que frenar los niveles de gases de efecto invernadero es un bien público mundial, mientras que seguir el ritmo de contaminación es un mal público mundial, porque los efectos de las externalidades se difunden indivisiblemente por todo el planeta. Esto quiere decir que es responsabilidad de todos, y al mismo tiempo obligación de nadie, poner fin al cambio climático. Por tanto, "ni los individuos, ni los mercados, ni los gobiernos nacionales tienen incentivos necesarios para encontrar un resultado eficiente". ${ }^{20}$ En este sentido, citando a Samuelson y Nordhaus, "las empresas no limitan voluntariamente las emisiones de sustancias químicas nocivas, ni se abstienen siempre de verter residuos tóxicos. Por lo que se considera que el control de la contaminación es una función legítima del Estado". ${ }^{21}$ Así, se pone en duda la funcionalidad de esta propuesta.

4. Con relación a obligar tanto a los países desarrollados como a los no desarrollados a imponer medidas para disminuir las emisiones de gases, resulta razonable, ya que muchos de los países en desarrollo contaminan más que los desarrollados. Sin embargo, recordemos algunos elementos de los países no desarrollados: 1. Los países de desarrollo bajo obtienen su principal fuente de ingreso del sector primario; es decir, tienen niveles de contaminación relativamente bajos. 2. En los países de desarrollo medio, la industria pesada (muy contaminante) es la de mayor contribución a PIB. Sin embargo, según la teoría de la moder- nización, estos países se encuentran en ese grado de desarrollo por un proceso natural evolutivo por el cual los países actualmente desarrollados también pasaron. Evidentemente, la infraestructura productiva de los países en vías de desarrollo no cuenta con tecnología de punta (menos contaminante) en sus sistemas productivos; ahora bien, importarla sería la solución, pero en este caso debemos considerar los incentivos que tiene la empresa para invertir en capital cuando ello representa encarecer el producto (con el fin de recuperar la inversión), cuando el mercado al que se ofrecen sus mercancías son en su mayoría de ingreso de medio y bajos, y además no existe en el país un marco legal (o es muy endeble) que obligue a la empresa a disminuir su contaminación. En este sentido, es muy complejo exigirles a los países no desarrollados las mismas condiciones de compromiso que a los países industrializados.

5. El énfasis de la AP6 en no imponer a sus socios obligaciones firmes para disminuir los gases de efecto invernadero sí marca, de manera formal, alguna diferencia con el Protocolo de Kyoto, pues este último determina que los países del anexo I están obligados a reducir sus emisiones, en el periodo 2008-2012, por lo menos en $5.2 \%$ con relación a los niveles de 1990 . Sin embargo, el Protocolo de Kyoto, como la gran mayoría de los regímenes internacionales, no cuenta con un gobierno con poder o autoridad para hacer cumplir los acuerdos. ${ }^{22}$ Así, la obligatoriedad de reducir sus emisiones queda, en realidad, dentro del compromiso moral.

6. Por último, con relación a la utilización de la energía nuclear y la captura de carbono para aminorar el efecto invernadero, existen dos observaciones: 1. "la energía nuclear quedó excluida del Protocolo de Kyoto y de sus mecanismos flexibles: a) por 
su incapacidad para hacer frente de forma eficaz al problema del cambio climático, y b) por ser una energía sucia y peligrosa (que produce irreversibles residuos radiactivos que permanecen durante decenas de miles de años"). ${ }^{23}$ 2. La captura de carbono no es viable porque proponen almacenarla bajo la tierra o los océanos, lo que conlleva demasiados riesgos ambientales, que se trasladan a las futuras generaciones.

En suma, la Asociación Asia Pacífico sobre el Desarrollo Limpio y Cambio Climático no ofrece propuestas novedosas o factibles, por lo que en lo hasta aquí desarrollado no se encuentran elementos para entender la decisión de Australia de crear esta nueva propuesta. Sin embargo, haciendo un análisis de las opiniones de los organismos no gubernamentales, encontramos elementos interesantes para comprender la postura de este país.

\section{La AP6, la opinión de las ONG y la conclusión}

Con posterioridad a la clausura de la cumbre, las críticas de los defensores del medio ambiente hacia la AP6 no se hicieron esperar. Cada uno de ellos, con sus aportaciones, agregó una pieza para terminar de armar el rompecabezas de este trabajo: la decisión de Australia de crear esta nueva propuesta.

Con relación a la utilización de energía nuclear, Ecoportal menciona que Australia y Estados Unidos incluyeron esta opción para fomentar la venta de uranio (para centrales nucleares) y de carbón, ambos recursos muy abundantes en Australia. ${ }^{24}$ Y agregó: "Este Pacto es un mero ejercicio de relaciones públicas dirigido a asegurar las ganancias de Australia y Estados Unidos.”

Por su parte, China y la India, ${ }^{25}$ países emergentes ávidos de energía nuclear, re- ciben con beneplácito esta disposición de venta "bajo fines ecológicos". En opinión de Greenpeace y los Amigos de la Tierra, las economías participantes utilizan la AP6 "como tapadera" para asegurarse el mercado del carbón en Asia. Mientras, Clive Hamilton, director del Instituto de Australia, concuerda con lo anterior al señalar "creo que en realidad esta conferencia sirve para proteger el futuro, a largo plazo, de la industria del carbón". ${ }^{26}$

En cuanto al uso de la tecnología para contrarrestar el incremento de emisiones de gases contaminantes, el Centro Pew Sobre el Cambio Climático Global señaló: "hay muy poco de nuevo en este pacto, que parece más bien un envase novedoso para iniciativas bilaterales y multilaterales de transferencia tecnológica ya existentes", y agregó: "la tecnología sola no es suficiente. Necesitamos incentivos a la industria y más intervención gubernamental". ${ }^{27}$ Mientras, Heikki Willstedt, experto en energía y cambio climático en wwF Adena, afirmó que los principales beneficiarios de esta asociación serían Japón, Estados Unidos y Australia, que venderán tecnología al resto de los estados miembros. ${ }^{28}$

Sobre la base de los elementos de los apartados anteriores, y a partir de la opinión de los expertos, se tienen los datos necesarios para determinar que las intenciones de Australia son más económicas que de protección al medio ambiente. Los argumentos de la Asociación Asia Pacífico sobre Desarrollo Limpio y Cambio Climático no se pueden sostener, mientras que las medidas propuestas están muy ad hoc con sus intereses.

En suma, es relativamente poco lo que Australia está dispuesta a ofrecer por disminuir el efecto invernadero y mantener un medio ambiente sano. Evidentemente, el Protocolo de Kyoto no es la panacea que 
va a poner fin al calentamiento de la Tierra, pues, en aras de perjudicar lo menos posible el sistema económico, el objetivo de alcanzar la reducción de emisiones de gases de efecto invernadero es pobre. No obstante, algo es mejor que nada. ny

\section{Notas}

1 Mural, en: http://www.mural/parseo/printpage. asp?categoriaid $=24 \& . . . /$ defaulr.htm.

2 Terra, "El acuerdo Asia-Pacífico de Cambio Climático concluye hoy con compromiso de erradicación del efecto invernadero" 2006, en: http://actualidad.terra.es/common/imprimir/ portada.cfm?id=AV2680745.

3 Radio Mundo Real, "Conversaciones sobre cambio climático anti-Kioto en Australia, en: http://www.radiomundoreal.fm/print.php?sid $=8217 \&$ POSTNUKESID=060eaa810d5af16.

4 Boicot a Kyoto, en: http://www.revistafusion. com/2005/octublre/ong145.htm.

5 EE.UU. y Australia lanzan una Asociación de Desarrollo Limpio con China, India, Japón y Corea del Sur, en: http://www.laraza.com.

6 El Protocolo de Kyoto, en: http://www.appa. es/dch/protocolo_kyoto.htm.

7 Alemania, Australia, Austria, Bélgica, Bielorrusia, Bulgaria, Canadá, Checoslovaquia, Dinamarca, La Comunidad Económica Europea, España, Estados Unidos, Estonia. Federación Rusa, Finlandia, Francia, Grecia, Holanda, Hungría, Irlanda, Islandia, Italia, Japón, Letonia, Lituania, Luxemburgo, Noruega, Nueva Zelanda, Polonia, Portugal, Reino Unido, Rumania, Suecia, Suiza, Turquía y Ucrania.

8 Inforesources focus, "Cambio climático, poblaciones rurales y recursos forestales", 2004, en: www.inforesources.ch.

$9 \quad$ Field Barry y Martha Field (2003), Economía ambiental, España, McGraw-Hill.

10 Un país desarrollado invierte en otro país desarrollado, en proyecto de energía limpia. El inversor obtiene un certificado para reducir sus emisiones a un precio menor al que le habría costado en su país.

11 Un país desarrollado invierte en tecnología de desarrollo limpio en un país en vías de desarrollo. El recorte de la contaminación derivado de esta inversión se documenta en certificado que la compañía puede intercambiar por derechos de emisión del país de origen.

12 Los países que emitan por debajo del límite impuesto por el Protocolo pueden vender sus emisiones a aquellos países que los excedan.

13 Gobiernos no pagaran por cambio climático, en http://www.portafolio.com.co/port_secc_online/ porta:_inte_online/2006-01-12/ARTICUL...

14 Conversaciones sobre cambio climático antiKyoto en Australia, en: 12 de Enero de 2006 http://www.radiomundoreal.fm/modules. php op $=$ modload $\&$ name $=$ News $\&$ file $=$ article $\&$ sid $=8217 \&$ mode=thread\&order $=0 \&$ thold $=0$.

15 Boicot a Kyoto, en: http://www.revistafusion. com/2005/octublre/ong145.htm.

16 EE.UU. y Australia prometen 127 millones de dólares para plan de reducción de gases de efecto invernadero, 2006, en: http://es.news.yahoo. com/12012006/4/cronica-clima-eeuu-australiaprometen-127 millones-dolares-plan-reduccion. html.

17 Consumer.es, La Alianza Asia Pacífico pretende discutir el próximo miércoles una alternativa al Protocolo de Kyoto, en: http://www.consumer. es/web/es/medio_ambiente/2006/01/09/148397. php?print=true.

18 EE.UU. y Australia prometen 127 millones de dólares para plan de reducción de gases de efecto invernadero, 2006, en: http://es.news.yahoo. com/12012006/4/cronica-clima-eeuu-australiaprometen-127 millones-dolares-plan-reduccion. html.

19 Consumer, es, La alianza Asia-Pacífico pretende discutir el próximo miércoles una alternativa al Protocolo de Kyoto, en: http://www.consumer. es/web/es/medio_ambiente/2006/01/09/148397. php?print=true.

20 Samuelson, Paul, y William Nordhaus, Economía (2002), decimoséptima edición, España, McGraw-Hill, p. 325.

21 Ibídem.

22 Greene, 2000:323.

23 Eco.Portal.Net, "Cambio climático: Greenpeace crítica a Estados Unidos y Australia por su nuevo intento de hundir el Protocolo de Kyoto, en: http://www.ecoportal.net/layout/set/print/ content/view/full/55635/.

24 Ibídem.

25 Bidwai, Praful, 2006, "El cambio climático es para todos", en: http://ipsnoticias.net/print. asp?idnews=36106.

26 Terra, Acuerdo Asia Pacífico de Cambio Climático concluye hoy con un compromiso de "erradicación del efecto invernadero", en: http:// wwwactualidad.terra.es/common/.

27 Leía, Stephen (2005), "Nuevo Pacto lejos de Kyoto, en: http://wwwtierramericana. net/2005/0806/noticias1.shtml.

28 Boicot a Kyoto, en: http://www.revistafusión. com/2005/octublre/ong145.htm.

MÉXICO YLACUENCA DEL PACÍFICO

vol. 8, núm. 25 / mayo-agosto de 2005 
The series Pacific Rim: Globalization, Regionalization, and Domestic Trajectories is composed

of four volumes, under the general supervision of Melba Falck and Arturo Santa-Cruz

\section{Volume 1. Globalization, Regionalization and Domestic Trajectories in the Pacific Rim: The Economic Impact}

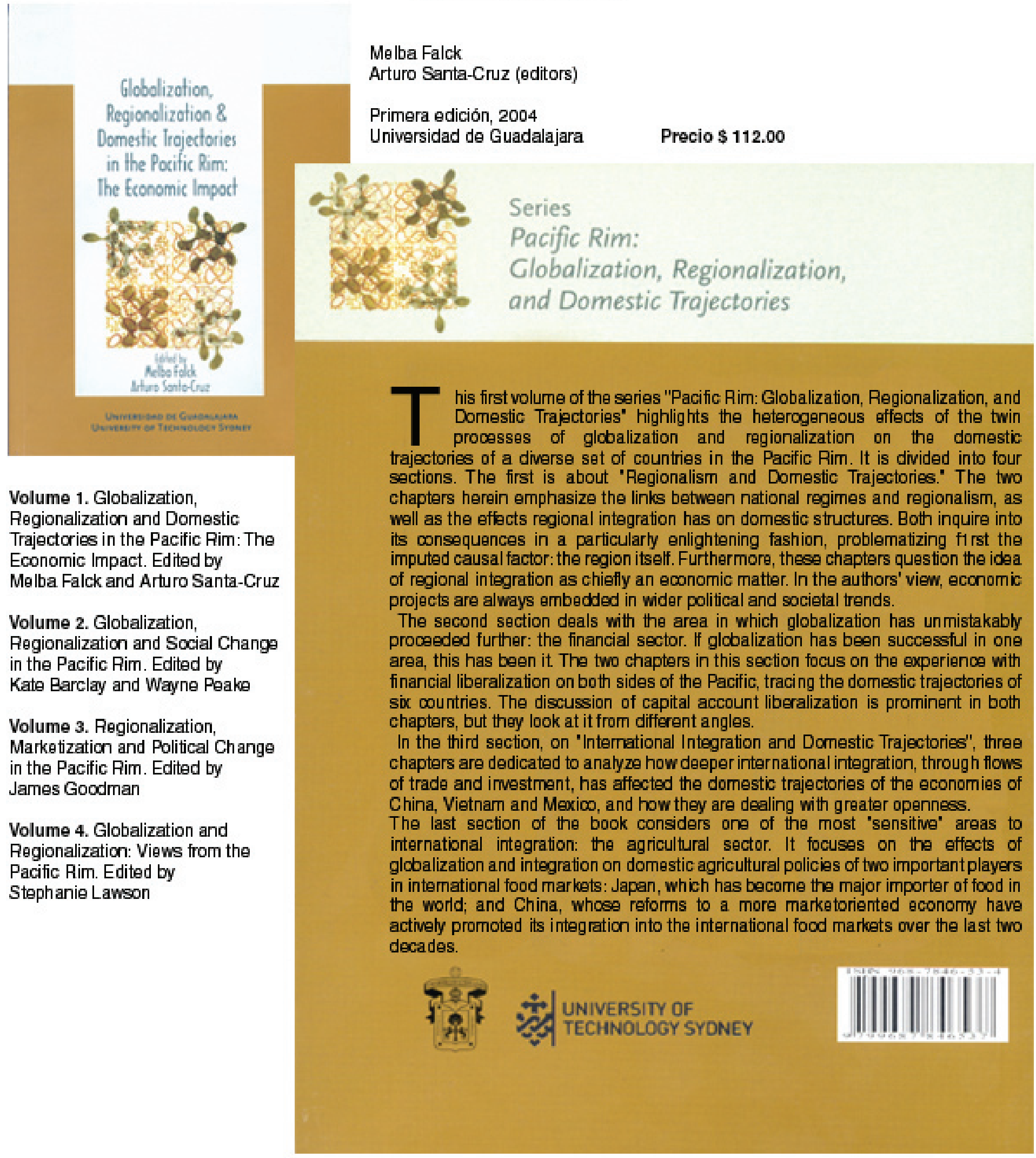

De venta en: librería el Kiosco, Centro Universitario de Ciencias Sociales y Humanidades, Av. Alcalde esquina Av. de los Maestros, edificio G, planta baja, Guadalajara, Jalisco 